\title{
Evaluation of container orchestration systems for deploying and managing NoSQL database clusters
}

Container orchestration (CO) systems, such as Docker Swarm, Kubernetes, Mesos Marathon and DC/OS, have been initially designed for stateless services. However, they have also been used for running database clusters due to improved automated management. This work evaluates the performance overhead of Docker Swarm and Kubernetes when running NoSQL database clusters, with MongoDB and Cassandra as case study.

https://github.com/eddytruyen/containers_on_openstack/blob/master/README.md

IEEE International Conference on Cloud Computing (IEEE Cloud 2018),

Workshop 2: Cloud Infrastructure, Tuesday July 3, 8:30am

\section{Common $\mathrm{CO}$ features for database clusters}
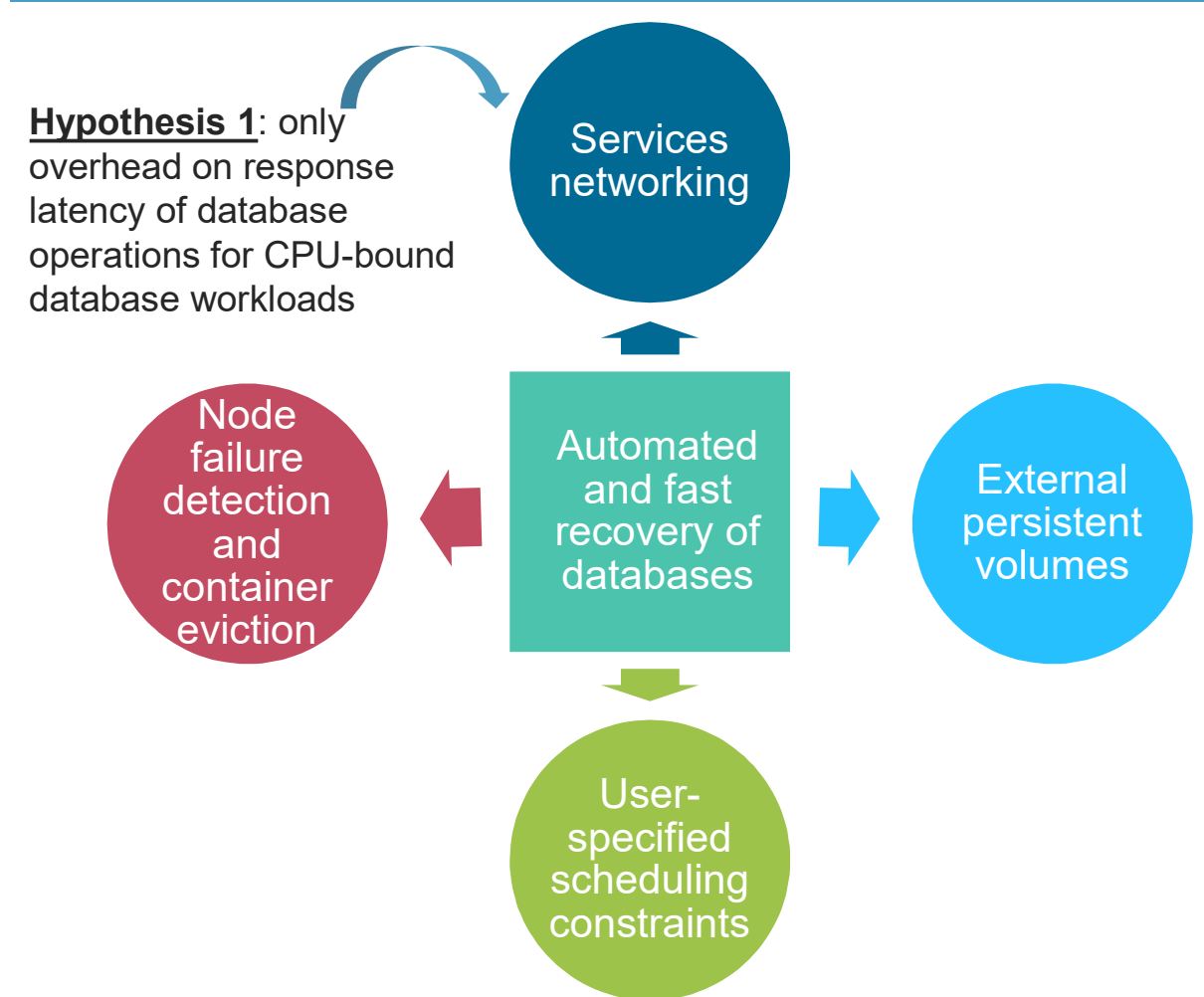

Hypothesis 2: no overhead on response latency for bottom 3 features

\section{Results for YCSB benchmark ${ }_{[1]}$}

\section{Hypothesis 1:}

For CPU-bound Cassandra workloads: services networking causes

overhead due to intermediate virtual network bridge in comparison to

DockerOnly without NAT (--net=host option)
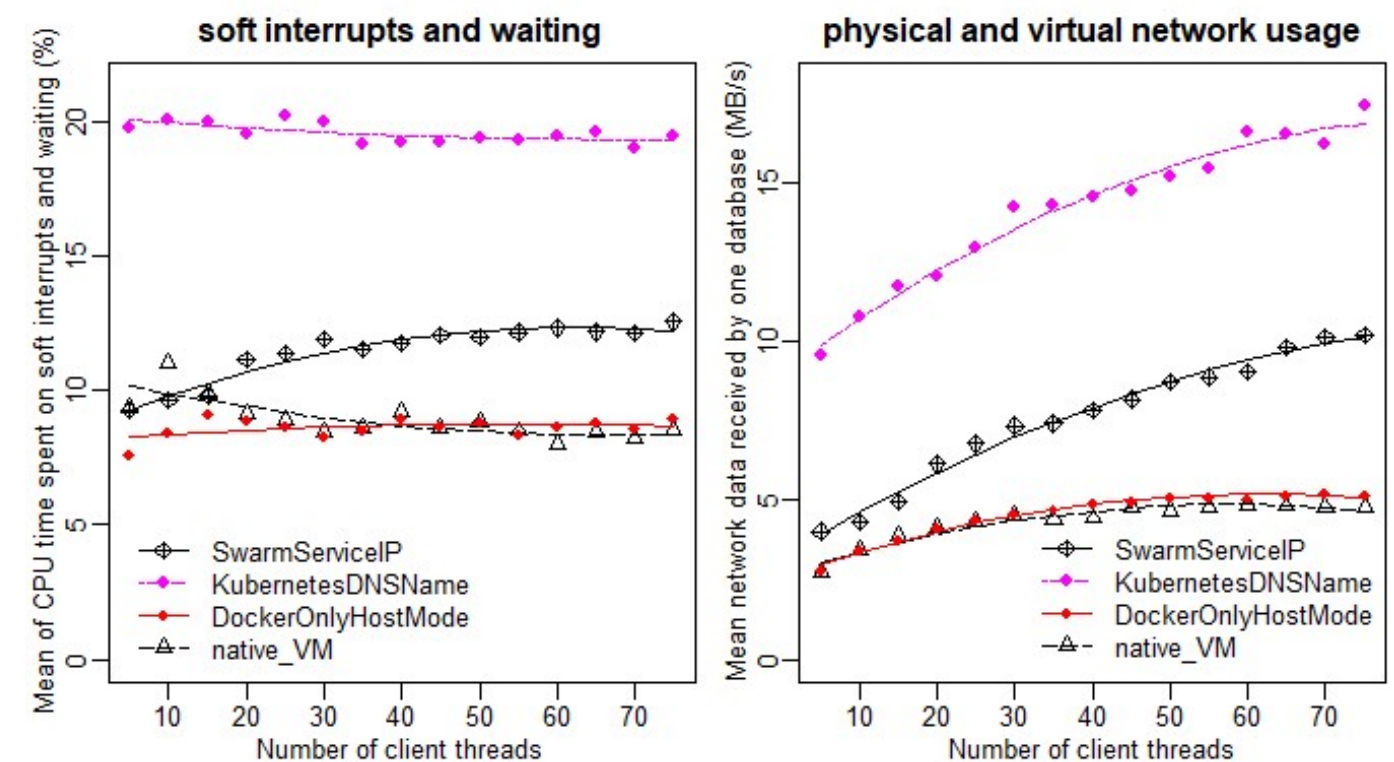

Resource usage graphs for Cassandra 2.0; 3-node cluster, Openstack VMs 2CPU 4GB, Ubuntu 16.04 YCSB workload A, $100 \%$ insert operations, size of dataset $=11.7 \mathrm{~GB}=>$ write-optimized database

For CPU-bound MongoDB workloads: Wrt the mean and $95^{\text {th }}$ quantile of response latency metrics, common rankings between services networking approaches are found for specific combinations of YCSB workload type and operation type:

\section{Three services networking approaches}

\begin{tabular}{|c|c|c|c|c|c|}
\hline $\begin{array}{l}\text { Service } \\
\text { networking } \\
\text { approach }\end{array}$ & Common features among $\mathrm{CO}$ systems & ह & 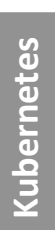 & $\begin{array}{l}\frac{5}{0} \\
\frac{2}{0} \\
\frac{0}{0} \\
\frac{0}{20}\end{array}$ & $\frac{\tilde{g}}{\mathrm{~g}}$ \\
\hline \multirow{2}{*}{$\begin{array}{l}\text { Routing mesh } \\
\text { with service } \\
\text { node ports }\end{array}$} & Distributed Layer 4 Load balancer (with ipvs) & $\checkmark$ & $\checkmark$ & & \\
\hline & Central L4-L7 load balancer (without ipvs) & $\checkmark$ & $\checkmark$ & $\checkmark$ & $\checkmark$ \\
\hline \multirow{3}{*}{$\begin{array}{l}\text { Virtual } \\
\text { network with } \\
\text { service IPs }\end{array}$} & Distributed Layer4 load balancer (with ipvs) & $\checkmark$ & $\checkmark$ & & $\checkmark$ \\
\hline & with stable DNS name for services & $\checkmark$ & $\checkmark$ & & $\checkmark$ \\
\hline & IP per container & $\checkmark$ & $\checkmark$ & $\checkmark$ & $\checkmark$ \\
\hline \multirow{3}{*}{$\begin{array}{l}\text { Host ports } \\
\text { networking } \\
\text { with VM IPs }\end{array}$} & Mapping container port to host port & $\checkmark$ & $\checkmark$ & $\checkmark$ & $\checkmark$ \\
\hline & Automated allocation of host ports & $\checkmark$ & & $\checkmark$ & $\checkmark$ \\
\hline & Static host port conflict management & $\checkmark$ & $\checkmark$ & & \\
\hline
\end{tabular}

\begin{tabular}{|c|c|c|}
\hline Operation & Update-heavy workloads $(\mathrm{A}, \mathrm{F})$ & Read-heavy workloads (B,C,D) \\
\hline Read & $\begin{array}{l}\text { 4.8, SwarmFloatingIPHostPort } \\
\text { 4.9,SwarmHostNameNodePort } \\
\text { 5.5,K8FloatingIPHostPort } \\
\text { 5.5,K8ServiceIP } \\
\text { 5.6,K8HostNameNodePort } \\
\text { 5.7,K8ContainerDNSName } \\
\text { 23.6,SwarmServiceIP }\end{array}$ & $\begin{array}{l}\text { 6.7,K8ServiceIP } \\
\text { 7.1,K8ContainerDNSName } \\
\text { 7.5,SwarmFloatingIPHostPort } \\
\text { 7.9,K8HostNameNodePort } \\
\text { 7.9,SwarmHostNameNodePort } \\
\text { 8.2,K8FloatingIPHostPort } \\
\text { 10.6,SwarmServiceIP }\end{array}$ \\
\hline $\begin{array}{l}\text { Update/ } \\
\text { Insert }\end{array}$ & $\begin{array}{l}\text { 20.2,SwarmFloatingIPHostPort } \\
\text { 21.1,K8FloatingIPHostPort } \\
\text { 21.3,K8ServiceIP } \\
\text { 21.8,K8HostNameNodePort } \\
\text { 22.2,K8ContainerDNSName } \\
\text { 23.8,SwarmHostNameNodePort } \\
\text { 43.8,SwarmServiceIP }\end{array}$ & $\begin{array}{l}\text { 18.9,K8HostNameNodePort } \\
\text { 19.1,K8FloatingIPHostPort } \\
\text { 20.2,SwarmFloatingIPHostPort } \\
\text { 21.3,SwarmHostNameNodePort } \\
\text { 26.1,K8ContainerDNSName } \\
\text { 27,5,K8ServiceIP } \\
\text { 37.5,SwarmServiceIP }\end{array}$ \\
\hline
\end{tabular}

95th quantile of response latencies for MongoDB 3.2.4; 3 node cluster, VMs 2CPU 4GB,Ubuntu 16.04; size of dataset = 23MB; => in-memory database

Hypothesis 2: For CPU-bound Cassandra workloads: in comparison to VMbased deployments, local volume plugins for Docker Swarm and Kubernetes are disk i/o performance bottlenecks. However, default volume drivers in DockerOnly deployments are not!

disk i/o bandwidth for write requests
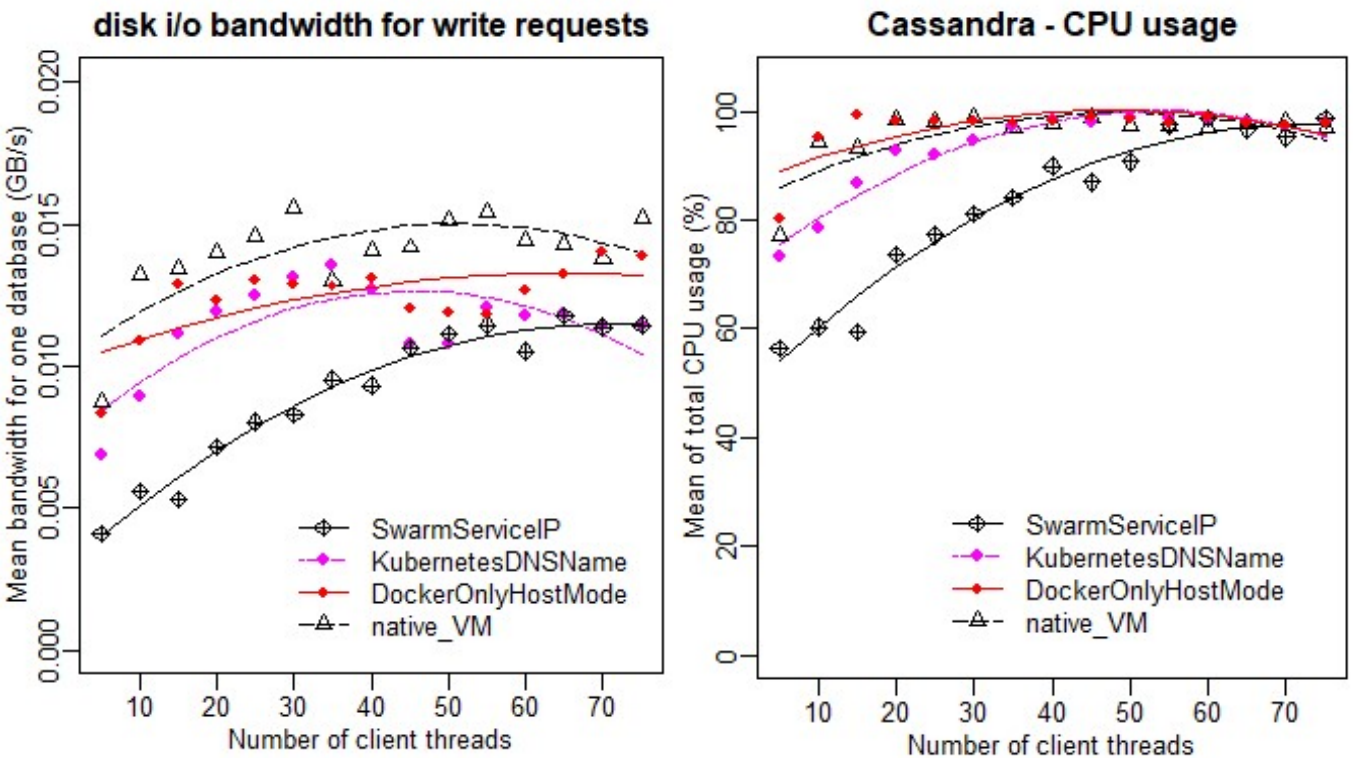